\title{
Discours
}

Revue de linguistique, psycholinguistique et

informatique. A journal of linguistics, psycholinguistics

and computational linguistics

9| 2011

Varia

\section{«This » comme marqueur privilégié du genre : le cas des résumés de thèses}

\section{Geneviève Bordet}

\section{OpenEdition}

Journals

Édition électronique

URL : http://journals.openedition.org/discours/8506

DOI : $10.4000 /$ discours.8506

ISSN : 1963-1723

Éditeur :

Laboratoire LATTICE, Presses universitaires de Caen

\section{Référence électronique}

Geneviève Bordet, « «This » comme marqueur privilégié du genre : le cas des résumés de thèses », Discours [En ligne], 9 | 2011, mis en ligne le 20 décembre 2011, consulté le 30 avril 2019. URL : http:// journals.openedition.org/discours/8506 ; DOI : 10.4000/discours.8506

\section{(c) (i) (3) $\Theta$}

Discours est mis à disposition selon les termes de la licence Creative Commons Attribution - Pas d'Utilisation Commerciale - Pas de Modification 4.0 International. 

Revue de linguistique, psycholinguistique et informatique

\section{"This » comme marqueur privilégié du genre: le cas des résumés de thèses}

Geneviève Bordet

Geneviève Bordet, «"This" comme marqueur privilégié du genre: le cas des résumés de thèses», Discours [En ligne], 9 | 2011, mis en ligne le 20 décembre 2011.

URL: http://discours.revues.org/8506. Consulté le 20 décembre 2011.

Titre du numéro: Varia

Coordination: Anne Le Draoulec et Josette Rebeyrolle 



\title{
«This » comme marqueur privilégié du genre : le cas des résumés de thèses
}

\author{
Geneviève Bordet
}

Les enjeux de la communication scientifique font de l'analyse du genre un objectif prioritaire, en tant que discours adapté aux objectifs de communication d'une communauté spécialisée. Cette recherche vise à comprendre comment l'utilisation d'un marqueur de référence tel que «this » en anglais, contribue à l'inscription du résumé de thèse dans un genre et à l'atteinte de ses objectifs. Nous postulons que «this» fait partie des outils rhétoriques adaptés à un discours caractérisé par sa brièveté et son importance symbolique dans le champ académique. À partir d'un corpus constitué de résumés écrits en anglais dans cinq disciplines par des locuteurs anglophones et francophones, nous proposons une typologie des fonctions de «this» qui créent la focalisation et définissent une stratégie rhétorique. Nous distinguons une fonction exophorique qui ancre le résumé dans son contexte d'énonciation, d'une fonction endophorique qui fait progresser le texte d'un mouvement rhétorique à l'autre, selon des modalités allant de la reprise au déplacement et à l'englobement. L'application de cette classification aux sous-corpus de didactique des mathématiques et de sciences des matériaux permet de dessiner diverses stratégies de communication. Nous montrons que I'utilisation pertinente de l'espace d'interprétation ouvert par «this» contribue à mettre en avant la légitimité et l'autorité du doctorant dans son domaine de spécialité, favorisant ainsi son admission dans la communauté scientifique.

Mots clés: analyse du genre, référence, résumé de thèse, exophore, anaphore, encapsulation, fonction rhétorique, discours spécialisé

The ever-increasing impact of academic publishing has made genre analysis a priority, since it defines the requirements of the communicative goals inside a specific community. This study aims at exploring how the reference marker "this" contributes to making the PhD abstract a recognizable type of genre and as such likely to fulfil its objectives. "This", as a rhetorical device, seems particularly adapted to a type of discourse characterized by its distinctive conciseness and highly symbolic status in the academic field. Our corpus includes abstracts from five disciplines, written in English by English and French-speaking doctoral students. This provides evidence for establishing a typology of the functions of "this" which create focalisation, and hence determine a rhetorical strategy. The exophoric "this" establishes the link between the abstract and its context of enunciation while its endophoric uses create the dynamics of the focus structure, ranging from a simple repetitive anaphor to a shift or encapsulation of the reference. The application of this classification to a corpus of abstracts in the fields of mathematics education and materials science highlights an array of rhetorical strategies depending on the writer's disciplinary and linguistic origin. We argue that the mastering of the interpretive space provided by the use of "this" helps the PhD candidate to demonstrate his/her legitimacy and authority, therefore contributing to his/her admission inside the academic community.

Keywords: genre analysis, reference, PhD abstract, exophoric use, anaphoric use, encapsulation, rhetorical function, specialized discourse

Langue étudiée: anglais

Marqueur linguistique: «this» 


\section{Introduction}

Depuis une vingtaine d'années, et dans un contexte de prolifération des types d'écrits, les études sur le discours se sont attachées à mieux comprendre la notion de genre, prise comme type de discours suscité par une situation de communication spécifique dans une communauté (Swales, 1990). Ces études de genre ont porté notamment sur le discours scientifique et académique, dont les modalités de diffusion se sont complexifiées tandis que les enjeux de publication se faisaient de plus en plus pesants. De très nombreuses études se sont attachées à mieux définir les règles externes qui régissent le genre et lui permettent de répondre aux objectifs de communication d'une communauté spécialisée (Swales, 1990; Hyland, 2000, 2002, notamment). Plus récemment, des spécialistes de l'analyse de discours et de la linguistique de corpus ont combiné leurs approches (Biber, Connor et Upton, 2007; Gledhill, 1994, par exemple) pour comprendre les mécanismes linguistiques internes qui inscrivent le texte dans un genre spécifique et en définissent le succès, ou l'échec, quant aux objectifs définis par la situation de communication.

Parmi ces mécanismes, un domaine de recherche s'est avéré particulièrement prolifique: celui des marqueurs de discours, éléments syntaxiques dont le choix et les modalités d'usage marquent une stratégie rhétorique au service des objectifs du genre. Ces marqueurs, qui incluent notamment démonstratifs, conjonctions et connecteurs logiques, ont pour fonction de marquer la progression logique et rhétorique d'un texte, garantissant les conditions d'une interprétation cohérente par le lecteur (Carter-Thomas, 2008).

Nous reprendrons ici la définition, souvent citée, des marqueurs du discours par Risselada et Spooren (I998: I32):

Discourse markers can be defined as those natural language expressions whose primary function is to facilitate the process of interpreting the coherence relation(s) between a particular unit of discourse and other, surrounding units and/or aspects of the communicative situation.

Risselada et Spooren mettent donc en évidence le lien entre l'interprétation de la cohérence du discours par un lecteur et l'adéquation de ce discours avec la situation de communication et ses objectifs contextuels. Nous nous attacherons particulièrement à élucider la manière dont ces marqueurs contribuent à la progression vers les objectifs de communication du genre.

Notre ambition est en effet, d'une part, de comprendre dans quelle mesure les différences de spécialités entraînent un maniement différent des stratégies rhétoriques et donc des marqueurs, et d'autre part, de vérifier notre hypothèse selon laquelle l'efficacité de l'inscription dans le genre passe autant par l'usage de marqueurs de la langue générale que par la maîtrise d'une langue de spécialité. Le genre auquel nous consacrons notre étude étant défini par la concision et l'absence de repères formels, on peut donc supposer qu'il est notamment caractérisé par le 
recours à des marques linguistiques aussi brèves et efficaces que possible. C'est dans ce contexte que nous avons choisi d'analyser la manière dont le démonstratif «this» contribue à créer un résumé de thèse conforme aux objectifs du genre et susceptible d'étayer la crédibilité du doctorant en tant que futur chercheur dans une discipline donnée.

Nous proposerons ainsi une typologie des différentes fonctions rhétoriques assurées par «this» dans ce type de résumé. Cette typologie devrait nous aider à mieux comprendre comment les variations dans l'utilisation de ces fonctions assurent l'efficacité cohésive de «this», puis la manière dont cette fonction cohésive participe d'une stratégie de communication qui contribue plus ou moins efficacement au succès de l'inscription d'un texte dans ce genre. Notre étude s'efforcera de prendre en compte les facteurs de variation tenant à la discipline concernée et à l'origine linguistique du locuteur, selon qu'il s'agit d'anglophones s'exprimant en langue native ou de francophones s'exprimant en langue seconde. Elle porte donc sur des résumés de thèse écrits en anglais par des locuteurs natifs de l'anglais et des locuteurs francophones, dans la même discipline et le même domaine de recherche. Notre objectif étant de mieux comprendre les facteurs d'efficacité d'une stratégie rhétorique liée à un genre, nous avons décidé, pour obtenir des données strictement comparables, de ne comparer que des textes rédigés en anglais par des locuteurs natifs et des locuteurs francophones. Les résumés français, que nous avons également collectés, comme nous le décrirons plus bas, restent ainsi à notre disposition pour une confirmation ultérieure de nos hypothèses quant aux effets des différences de maîtrise linguistique sur les écarts de stratégie discursive constatés, en anglais, entre locuteurs de Lr et de L2. Ceci permettrait d'enrichir les perspectives didactiques sur lesquelles débouche cette étude, en matière d'aide à la rédaction scientifique, en seconde langue notamment.

\section{Cadre théorique des études sur le genre}

\subsection{Le genre comme norme collective et stratégie individuelle}

Nous nous situons dans la perspective de Swales (1990), qui considère le genre comme un type de discours lié à une communauté et des objectifs de discours spécifiques, mais aussi dans celle de Rastier (2005). Celui-ci pointe le fait que le genre doit, au niveau du corpus, se conformer à la norme implicite définie par l'attente de la communauté de discours, tout en intégrant les variations introduites, au niveau du texte, par chaque réalisation individuelle. Autrement dit, c'est à travers l'espace laissé aux variations des stratégies rhétoriques particulières que se dessine l'horizon d'attente commun et implicite de la communauté de discours.

Dans cette perspective, l'étude des résumés de thèses nous paraît présenter un cas particulièrement intéressant dans la mesure où il s'agit d'un type de discours lié à une situation institutionnelle très spécifique, donc d'un genre régi par des objectifs de communication clairement identifiables. 


\subsection{Les résumés de thèses en tant que genre}

Le choix des résumés de thèses comme corpus d'application d'une recherche peut en effet surprendre pour plusieurs raisons. Tout d'abord, ils représentent un enjeu stratégique beaucoup moins important que le résumé d'article ou de communication, lesquels interviennent de manière décisive en amont et en aval du processus de recherche lui-même. En effet, c'est la plupart du temps sur la foi d'un résumé qu'une communication ou un article sont acceptés ou rejetés. Mais c'est aussi, après la publication, grâce au résumé que sont repérés, sur Internet, articles et communications, présentés maintenant dans des bases de données spécialisées telles que Scopus ${ }^{1}$ notamment. L'explosion du nombre des publications, la mondialisation et la dématérialisation des canaux de publication, ont rendu vitale la lutte pour la visibilité chez les chercheurs: il ne suffit plus de publier, il faut encore le faire savoir.

La plus grande partie des études du discours scientifique et académique porte donc logiquement sur l'article scientifique et son résumé. Et si toutes sortes de discours académiques écrits et oraux ont été pris en compte (Biber, 2006) jusqu'aux textes de remerciements (Hyland, 2004), les résumés de thèses ont été l'objet de peu d'attention, comme le note Swales (1990: 18I), à l'exception de Holzem (2000) qui a analysé la reformulation dans le résumé de thèse en français et de Swales et Feak (2009) qui en ont proposé une approche didactique. Les résumés de thèses, accessibles depuis longtemps via des serveurs spécialisés tels que Scirus ETD ${ }^{2}$ pour le domaine anglo-saxon, ont récemment été mis en valeur en France grâce au développement par le CNRS du serveur TEL ${ }^{3}$, qui en propose systématiquement une version bilingue français-anglais.

Au-delà de sa visibilité, en plein développement, nous nous sommes surtout intéressée au résumé de thèse en tant que représentation miniaturisée et ambassadeur de cette pierre de touche de l'entrée dans le monde de la recherche que constitue la thèse, dans l'univers francophone comme anglo-saxon. Après Swales (I990) parlant de «gatekeeper» et Hyland (2000) qualifiant le doctorant de «would-be insider», nous proposons de considérer la thèse comme l'épreuve initiatique qui préside à l'admission dans la communauté scientifique de la recherche. Comme Hyland (2000) toujours, nous proposons de traiter le résumé comme l'outil autopromotionnel non seulement d'un écrit mais de son auteur lui-même. Une des spécificités du résumé de thèse est de devoir représenter en quelques mots, dont le nombre est souvent imposé, une recherche menée sur plusieurs années et son produit, qui atteint souvent plusieurs centaines de pages. Il représente donc pour le candidat un effort de distanciation particulièrement difficile, mais c'est aussi le type de performance qu'il devra réaliser tout au long de sa carrière de chercheur pour faire accepter ses propositions d'articles et de communications.

1. Base de données Scopus: http://www.scopus.com/home.url.

2. Formulaire de recherche sur Scirus ETD: http://www.ndltd.org/serviceproviders/scirus-etd-search.

3. Serveur TEL (thèses-en-ligne) : http://tel.archives-ouvertes.fr/. 
Au-delà de la recherche et de son produit, et puisque la thèse constitue l'acte de candidature d'une personne, c'est sa personnalité et ses qualités de chercheur que l'auteur du résumé de thèse cherchera à mettre en valeur. Il devra particulièrement, à travers la présentation de son travail, mettre en évidence sa capacité à construire une cohérence, à prouver sa légitimité dans sa discipline, et à faire preuve d'autorité en apportant une contribution nouvelle à son domaine de recherche (Hyland, 2000 et 2002). Ce sont ces trois qualités qui feront de lui un «aspirant-chercheur» susceptible d'être admis dans la communauté de la recherche. Nous verrons plus loin que, dans une certaine mesure, ces qualités se déclinent différemment selon la discipline concernée. Leur mise en évidence constitue néanmoins l'objectif commun du genre que constitue le résumé de thèse, caractérisé par une situation institutionnelle fortement marquée.

Par ailleurs, et comme nous l'avons noté, la forme extrêmement courte du résumé de thèse par rapport au document qu'il représente accentue la difficulté de sa réalisation. Ce texte court présente en effet très peu ou pas de marques formelles de structuration, telles que sous-titres ou marques de paragraphe. Cette absence de repères formels a pour conséquence la nécessité de recourir à des marques linguistiques, qui doivent pourtant être aussi brèves et efficaces que possible. Le résumé de thèse devant rendre compte d'un processus long et complexe, il devra, avec une économie de moyens rhétoriques remarquable, construire une représentation crédible et convaincante du scénario de la recherche qui a été menée. Ceci implique une série de déplacements très rapides de la focalisation de l'attention du lecteur, focalisation qui soutient le lecteur dans sa construction d'une représentation conceptuelle du travail de recherche réalisé. D'où l'importance du choix de chaque terme et notamment des termes susceptibles de construire la cohérence du texte, en assurant le passage d'un point de focalisation conceptuelle à un autre.

C'est dans ce contexte que nous avons choisi d'étudier plus particulièrement le fonctionnement, dans ce genre spécifique, du marqueur de discours «this».

\section{3. «This» comme marqueur du genre}

Les critères qui ont présidé au choix de ce marqueur sont d'ordre quantitatif et qualitatif. Une première étude statistique de notre corpus de résumés de thèses, décrit plus bas, fait en effet apparaittre sa récurrence particulièrement frappante.

Ainsi, «this» apparaît en $\mathrm{II}^{\mathrm{e}}$ position, juste après les articles déterminés et indéterminés «the» et «a», la conjonction «and», les prépositions les plus courantes «of», «to», «in», le relatif ou déterminant «that», le verbe «is» et le pronom personnel «we». Compte tenu de la fréquence habituelle de ces termes, il s'agit d'un rang très élevé. À titre de comparaison, le marqueur de référence le plus fréquent «it» n'arrive qu'en $28^{\mathrm{e}}$ position. La fréquence de «this» est d'autant plus frappante si on le compare cette fois avec les connecteurs cités par Hyland comme «textual metadiscourse logical connectives» (2000: 190). Des termes aussi habituellement courants que «because», «but», «besides» et «consequently» n'apparaissent qu'entre o et 4 fois 
pour chacun des sous-corpus analysés, dont nous précisons qu'ils comprennent Is textes d'environ une page; ceci à l'exception de «however», utilisé 7 fois dans un seul sous-corpus (voir plus loin: «3.I. Constitution du corpus»). Ce dernier point pourrait justifier une analyse particulière.

L'autre caractéristique intéressante de «this» est sa plasticité remarquable, relevée et analysée déjà par Halliday et Hasan (1976). Ce «self-reference segment» (Sinclair, 2005: 167) a en effet le pouvoir, avec une économie de moyens maximale, de déplacer l'attention du lecteur d'un point à l'autre du raisonnement (Charles, 2007) selon des modalités variées et avec une flexibilité remarquable comme nous le montrerons dans la typologie que nous proposons plus bas. Nous nous intéresserons particulièrement à cette capacité de déplacement de la focalisation en lien avec la place de «this» dans les mouvements rhétoriques successifs du texte (Apotheloz, 1995). Gledhill (1997) souligne également son pouvoir de «reformulation textuelle», lui permettant de reprendre et de transformer notamment un pan entier du texte.

Bien entendu, «this» ne constitue qu'un des dispositifs rhétoriques susceptibles de soutenir l'attention du lecteur et de le guider dans la progression de l'argumentation. Nous aborderons d'ailleurs dans notre conclusion les perspectives ouvertes par l'application de notre démarche à d'autres types de marqueurs de discours liés à l'expression de la référence, tels que «the» ou «it» par exemple.

\section{Cadre méthodologique}

\subsection{Constitution du corpus}

Comme nous l'avons déjà mentionné, notre recherche a porté sur un corpus de résumés de thèses rédigés en anglais par des locuteurs anglophones et francophones, dans 5 disciplines.

Nous avons sélectionné ces disciplines et domaines de recherche dans le monde des sciences dures, ce qui paraissait garantir un niveau de réalisation en anglais par des francophones d'une qualité suffisante pour que la stratégie discursive soit clairement discernable. Notre travail de collecte a en effet montré que dans certaines sciences humaines, notamment les sciences de l'éducation, la réalisation en anglais était si pauvre qu'elle aurait pu fausser toute interprétation des données recueillies.

Les disciplines ou domaines traités sont donc les suivants: sciences des matériaux, didactique des mathématiques, biologie cellulaire, algèbre mathématique, web sémantique. Notre corpus total comprend 48616 mots. Seuls les deux premiers domaines feront ici l'objet d'une analyse systématique, soit les résumés rédigés en anglais par des anglophones en didactique des mathématiques (DM LI) et en sciences des matériaux ( $\mathrm{SM} \mathrm{LI})$, et les résumés rédigés en anglais par des francophones en didactique des mathématiques (DM L2) et en sciences des matériaux (SM L2). Ils sont donc strictement comparables. Ces 4 sous-corpus comprennent chacun I5 textes et 20443 mots. 


\subsection{Une démarche d'analyse combinant analyse du texte et du corpus}

La taille relativement réduite de notre échantillon garantit la possibilité d'une double démarche d'analyse: au niveau du texte et au niveau du corpus. Dans la lignée de Biber, Connor et Upton (2007) notamment, il nous a paru en effet indispensable de pouvoir procéder à un aller-retour permettant de partir de la compréhension des mécanismes rhétoriques internes au texte pour procéder à une identification des tendances dans les stratégies adoptées au niveau du sous-corpus. Ces tendances ne seront cependant pas interprétées pour en tirer des conclusions définitives sur les caractéristiques identitaires d'une discipline par exemple; en revanche, elles permettront d'affiner les critères dont l'étude statistique appliquée à un corpus plus important permettrait de dessiner les stratégies et les valeurs mises en avant pour ce type d'écrit dans une discipline spécifique. C’est le cas, comme nous le montrerons plus loin, du choix opéré dans l'éventail ouvert par les différentes fonctions rhétoriques de «this».

\subsection{Détermination des mouvements rhétoriques}

L'ensemble des textes recueillis a été étiqueté en fonction des mouvements rhétoriques déterminés sur des critères sémantiques. En nous appuyant sur le modèle initialement défini par Swales (1990) et adapté pour les résumés d'articles par Hyland (2004), nous avons établi une typologie de quatre mouvements prototypiques: contextualisation institutionnelle ou théorique de la recherche, proposition de recherche, description de la méthode, et enfin résultats et perspectives.

\section{Analyse des données : établissement d'une typologie des fonctions de «this"}

Ce marquage des mouvements rhétoriques et le relevé systématique des occurrences de «this» en contexte, grâce à l'utilisation du concordancier Antconc ${ }^{4}$, ont abouti à l'établissement d'une typologie des fonctions de «this» que nous présenterons ci-dessous. Nous nous sommes largement appuyée pour cela sur la discussion de la notion d'anaphore par Apotheloz (I995). Nous reprenons sa proposition de considérer l'anaphore comme un élément déterminant de la construction de la cohérence d'un texte, en tant qu' «ensemble de dispositifs contribuant à assurer au texte sa progression» et «dispositif intimement lié à la dynamique textuelle» (Apotheloz, 1995: II). Ceci nous inscrit dans une perspective fonctionnelle. L'objet de notre recherche n'était en effet pas d'approfondir les mécanismes d'établissement de la référence, mais de proposer des catégories pragmatiques mettant en évidence la manière dont ce marqueur contribue à faire progresser le texte au long des mouvements rhétoriques.

L'utilisation de cette typologie nous permettra dans un second temps d'émettre des hypothèses quant au type de stratégie rhétorique mis en œuvre pour ce genre d'écrit dans une discipline donnée.

4. Concordancier Antconc: http://www.antlab.sci.waseda.ac.jp/software.html. 
Un premier niveau d'analyse du corpus a permis d'établir une distinction entre deux grandes fonctions de «this»: dans le premier cas, «this» et le terme qu'il détermine renvoient au document primaire que constitue la thèse comme dans l'exemple suivant:

[I] [Proposition] This thesis addresses the internationally recognised problem of transforming classroom teaching that is underpinned by transmissionist epistemologies, especially in the field of mathematics education [Proposition].

(DM LI)

Dans le second cas, «this» renvoie à un élément interne au résumé lui-même et procède donc à une opération de référence intratextuelle.

Nous examinerons successivement ces deux configurations et leurs déclinaisons.

\section{1. «This» exophorique}

Le cas de la référence externe appelle plusieurs commentaires. Tout d'abord, nous avons choisi le terme «exophorique» pour désigner la référence au monde réel lié à la situation de communication, à savoir la production d'un mémoire de recherche, en tant qu'à la fois processus et résultat. On pourrait également considérer ce lien comme déictique, pointant vers la situation de communication. Nous reprenons plus particulièrement ici le point de vue de Halliday et Hasan qui désignent comme exophorique le lien de «situational reference» (Halliday et Hasan, 1976: 33).

Notre corpus montre que le résumé de thèse dispose, pour exprimer cette fonction, d'une palette de termes relativement fermée : «study», «research», «thesis», «work» essentiellement.

Cependant, l'interprétation de la référence est plus complexe qu'il n'y paraît. En effet, en fonction de l'ensemble de l'environnement syntaxique du terme déterminé par «this», le lien peut être ainsi établi avec la thèse comme œuvre réalisée, ou avec le projet de recherche tel qu'il a été mené, ou encore avec le doctorant qui a mené le projet de recherche et rédigé la thèse.

Ainsi, dans l'exemple ci-dessus, l'usage du verbe «address» permet de concilier l'établissement d'un lien avec la thèse comme œuvre et la mise en place d'un lien avec l'auteur de la recherche, voire avec le processus de recherche lui-même. La présence de modalisants («internationally recognised», «especially») renforce le lien avec l'auteur lui-même, conciliant l'autorité de l'œuvre constituée avec la mise en valeur du point de vue de son auteur.

On comparera avec l'effet produit par l'exemple suivant:

[2] [Proposition] This study was conducted to analyse aspects of mental computation within primary school mathematics curricula and to formulate recommendations to 
inform future revisions to the Number strand of mathematics syllabuses for primary schools [Proposition].

(DM LI)

Le choix du terme déterminé («study»), le verbe («conduct»), la voix passive et le prétérit mettent en valeur le processus de recherche, la part de l'auteur étant exprimée en deuxième niveau, dans la relative, avec le choix de verbes («analyse», «formulate», «inform») fortement interprétatifs.

C'est donc l'ensemble de l'environnement syntaxico-sémantique de «this» qui permet à ce marqueur non seulement d'établir un lien avec l'objet représenté, mais aussi d'amorcer un scénario de présentation de la thèse, selon trois possibilités: étayer sa légitimité sur l'œuvre réalisée («this thesis presents»), mettre en avant le dynamisme de la recherche («this study was conducted») ou valoriser la qualité de la contribution intellectuelle du chercheur, ce que l'auteur parvient également à faire dans les deux exemples présentés ci-dessus.

Nous proposons donc de qualifier ce type de lien d'«exophorique», considérant que le lien est établi ici avec le contexte situationnel que constitue la thèse comme document primaire mais aussi, comme nous l'avons vu, avec le processus de recherche et son auteur. L'antécédent de «this» n'est donc pas présent dans le texte du résumé, mais il est implicite et identifié par le lecteur dans le contexte d'un genre spécifique, celui du résumé de thèse. L'utilisation de «this» en contexte permet d'établir un lien solide avec le texte dont le résumé fait la promotion. Mais la manière dont le lien est établi par l'environnement syntaxique de «this» permet de maintenir un niveau d'ambiguïté suffisant pour pouvoir englober l'auteur et sa recherche, considérée comme processus ou résultat, selon la stratégie adoptée pour valoriser la thèse.

Il semble donc que ce cas particulier apporte une nouvelle contribution au débat portant sur la distinction entre anaphore, deixis et exophore (Cornish, 2010), et la nature de la distinction entre contexte externe, interne et situationnel. La discussion doit également prendre en compte les conditions de publication du résumé et son accessibilité. L'évolution des conditions d'accès à la thèse a un effet direct sur l'interprétation de cette fonction particulière de «this». Dans le contexte d'une publication imprimée, le résumé, lorsqu'il est lu en tête de la thèse, fait de la mention de type «this thesis» une référence cataphorique renvoyant au texte intégral de la thèse situé quelques pages plus loin. On peut dans ce cas parler de référence intratextuelle, le texte étant constitué par l'ensemble du document. Mais dans le contexte le plus courant actuellement, à savoir un résumé accessible en ligne et renvoyant à un texte de thèse accessible ou non, le plus souvent au format PDF, on a bien affaire physiquement à deux documents différenciés. C'est sur cette distinction que nous fondons notre choix du terme "exophorique», renvoyant à la fois au document primaire et aux conditions de sa production, dont nous postulons que l'ensemble constitue la situation d'énonciation liée au genre. 


\section{2. «This» endophorique}

[3] [Méthode] Finally, a new method to solve the cohesive crack problem in three
dimensions is proposed [Méthode_fin]. [Résultats_début] This method is capable of

[Méthode] Finally, a new method to solve the cohesive crack problem in three
dimensions is proposed [Méthode_fin]. [Résultats_début] This method is capable of

Nous traiterons maintenant du deuxième cas, celui où «this» fait référence à un élément interne du texte du résumé lui-même, fonction que nous qualifierons d'«endophorique», toujours en référence aux propositions de Halliday et Hasan (1976), et à leur discussion par Apotheloz (1995). Le relevé que nous avons mis en place et son analyse nous ont permis d'effectuer une distinction entre trois cas de figure, en fonction des modalités d'établissement de cette référence interne.

Dans le cas particulier du résumé de thèse, on remarquera que références exo- et endophoriques sont souvent liées, dans la mesure où le texte secondaire opère un choix parmi les termes centraux du document primaire: le fait de les reprendre et de les intégrer dans le résumé leur confère un statut de mots clés et en fait des références externes en même temps qu'internes, établissant le lien avec la thèse et avec ses référents théoriques.

D'un point de vue pragmatique, la fonction endophorique de «this» dans les résumés de thèses que nous avons étudiés se décline de la manière suivante:

- «this» et le terme qu'il détermine reprend un terme précédemment utilisé dans le texte;

- «this» et le terme qu'il détermine reprend non pas un terme mais un élément d'information précédemment présenté dans le texte;

- «this» utilisé comme pronom: il reprend également un élément d'information précédent mais sans y substituer un terme déterminé comme dans le cas ci-dessus.

Nous proposons ci-dessous une dénomination pour chacune de ces fonctions endophoriques, qui sont accompagnées de quelques exemples pour mieux comprendre ce qui les caractérise et leur influence spécifique sur la construction de la cohérence du texte et de la crédibilité de son auteur.

\section{3. «This» anaphorique de reprise}

Nous proposons de désigner comme "anaphorique de reprise» le cas d'une référence endophorique où «this» et son déterminé reprennent un terme à l'identique. Il s'agit ici de ce que l'on appelle également l'anaphore «fidèle», qui reprend le terme exact de la référence, par opposition avec l'anaphore «infidèle» qui le reprend indirectement.

Nous verrons que si le terme déterminé est une simple reprise, les variations entre l'environnement syntaxique du terme déterminé et celui du terme référent définissent la nature du changement de focalisation.

Voici un exemple d'utilisation anaphorique de reprise: 
modeling the propagation of both the true crack and process zone on a pre-defined crack path for different specimen geometries and absolute sizes [Résultats_fin].

(SM LI)

Le terme «method» figure dans la proposition immédiatement précédente et se trouve donc clairement identifiable. La reprise permet de faire progresser le raisonnement de la description de la méthode à la présentation des résultats. On notera la manière dont «this» porte la focalisation sur le caractère nouveau de l'objet introduit et ses potentialités.

Cet autre exemple témoigne d'une manière similaire de faire progresser le raisonnement en utilisant la même fonction de «this»:

[4] [Méthode] The physical origin of mathematical abilities and activities was one of the factors used in this study to establish an isomorphism between the nature and practice of mathematics with that of rituals [Méthode_fin]. [Résultats_début] This isomorphism provides the teaching and learning of mathematics with a more robust framework that is more attuned to the social nature of human beings [Résultats_fin]. (DM LI)

La reprise de «isomorphism» permet de poser comme établi un fait qui n'était encore qu'un postulat à l'étape précédente et de placer la focalisation sur un outil d'analyse du domaine.

Enfin, dans ce dernier exemple,

[5] [Méthode] A new, integrated, arbitrary, cohesive crack propagation strategy is proposed. This strategy is based on: interactive, effective total crack (true crack plus fracture process zone) length control [Méthode].

(SM LI)

la reprise de «strategy» ne marque pas un changement de thématisation mais permet d'attirer l'attention sur la liste des qualités mentionnées dans le référent, assurant une fonction de renforcement très efficace à l'intérieur d'un même mouvement rhétorique.

«This» anaphorique détermine très souvent un terme de langue scientifique générale (Pecman, 2007; Tutin, 2007) («strategy», «method») qui occupe une place centrale dans la discipline concernée, ici les sciences des matériaux. C'est l'environnement de ce terme général qui le transforme en collocation spécialisée, comme on le voit dans l'exemple ci-dessus.

\section{4. «This» anaphorique «shifter»}

Analysons maintenant le cas où «this» et son déterminé ne reprennent pas directement un terme précédent mais un élément d'information dont les contours restent à déterminer. 
En voici un exemple:

[6] [Résultats] Relatively minor changes in the specified crack velocity result in significantly different $\mathrm{KID}(\mathrm{V})$ curves. Therefore, extreme caution should be exercised when using this type of generated data [Résultats].

(SM LI)

L'opération de référence ouvre ici un espace d'interprétation. Le référent peut être uniquement la collocation «significantly different $\mathrm{KID}(\mathrm{V})$ curves», mais on peut également considérer que la référence englobe toute la phrase précédente, «this type of generated data» faisant référence au résultat du processus décrit dans la phrase.

On notera que, pour être correctement interprétée, la référence, dans ce cas, et parce qu'elle établit un lien entre deux collocations spécialisées, fait appel à des connaissances disciplinaires, associant de fait le lecteur à un champ de références supposées communes. Le lien entre élément déterminé et référent est, du point de vue terminologique, de type hyperonymique. Ceci ne peut que contribuer au renforcement de la légitimité disciplinaire du doctorant auteur du résumé.

On retrouve le même type de phénomène dans le corpus de didactique des mathématiques:

[7] [Contexte] Several research studies have confirmed that high-school and undergraduate students have a very poor knowledge of logarithms and logarithmic functions. One of the possible reasons for students' difficulties could be an insufficient teachers' knowledge of this subject domain [Contexte].

(DM LI)

Ici, le terme «this subject domain» opère un déplacement permettant de passer de la notion mathématique au domaine de la didactique spécialisée.

Ce troisième exemple est également extrait du corpus de didactique des mathématiques langue I:

[8] [Contexte_début] After an analysis of the School Certificate examination results for the past ten years in Mauritius, it was found that boys were out-performing girls in mathematics at that level [Contexte_fin]. [Proposition_début] This study aimed to examine this gender difference in mathematics performance at the secondary level [Proposition_fin].

(DM LI)

«This gender difference» reprend clairement l'affirmation «boys were outperforming girls» en la résumant. On retrouve ici le phénomène de nominalisation décrit par Halliday (2004) comme fondement de la construction de l'autorité scientifique. En requalifiant le phénomène décrit précédemment, ce candidat contribue nettement à asseoir son autorité en affichant sa capacité à utiliser la catégorie conceptuelle «gender difference». 
On voit donc que ce type d'utilisation de «this» déterminant réalise une double opération de déplacement et d'encapsulation. On pourrait parler d'«anaphore infidèle» dans la mesure où le terme déterminé ne reprend pas le terme exact de la référence, mais il s'agit ici du cas plus particulier où l'anaphore recatégorise et/ou résume le segment référentiel, ce qui correspond plutôt à la catégorie de l'anaphore conceptuelle ou résomptive.

Nous préférons cependant reprendre le terme de «shift» proposé par Francis (1994), qui met en valeur le déplacement opéré, et proposerons donc le terme plus dynamique de «shifter». On pourrait également envisager d'établir une distinction entre l'exemple de «this gender difference» qui allie déplacement et encapsulation d'une proposition entière, et celui de «this subject domain» qui opère un déplacement mais pas une encapsulation en ne reprenant que deux termes combinés. Toutefois, il nous semble que la différence est ténue et ne justifie pas la création d'une nouvelle catégorie. De plus, l'exemple précédent, reliant «KID curves» et «this type of generated data» montre que la portée exacte du déplacement peut être difficile à définir, ce qui ne compromet pas, bien au contraire, l'efficacité du procédé.

Ce n'est pas le cas dans ce dernier exemple extrait du corpus de sciences des matériaux rédigé par un locuteur francophone:

[9] [Méthode] [...] A modified Murakami criterion is proposed for such materials and the microstructural part of the anisotropy is also studied. In the same time we determine how to model the grain flow orientation in the numerical software Forge ${ }^{\circledR}$, which is devoted to the simulation of forming process [Méthode]. [Résultats] This new variable must be used afterwards to calculate the modified criterion [Résultats].

(SM L2)

Le référent de «this new variable» est difficile à identifier. À première lecture, le modalisant «new» semble renvoyer à «modified Murakami criterion» qui indique l'introduction d'une nouveauté. Cependant, cette interprétation est démentie par la suite, puisque c'est la «nouvelle variable» qui est utilisée pour calculer le «critère modifié». Une deuxième lecture permet d'émettre l'hypothèse que "new variable» se réfère de manière implicite au résultat de la modélisation de «l'orientation de l'écoulement de grains». Cette modélisation aurait permis la prise en compte d'une «nouvelle variable», que l'auteur suggère d'utiliser pour calculer le «critère modifié». Le terme «variable» renverrait donc à la mesure de l'écoulement, résultat du modèle élaboré. L'utilisation de ce terme contraint ainsi le lecteur à une sorte de «saut cognitif», certes plus accessible pour le lecteur spécialiste, mais cependant problématique. L'utilisation de «new» dans l'anaphore qui semble, sur un plan lexical, renvoyer à «modified» amène donc le lecteur à une première hypothèse interprétative erronée; le passage seulement implicite de la modélisation du «grain flow orientation» à la définition d'une «nouvelle variable» achève de brouiller l'opération de référence, que seule la maitrise du domaine de spécialité rend interprétable. On voit donc que les modalités de l'association entre termes de la langue scientifique générale (ici 
«criterion» et «variable») et termes spécialisés («grain flow orientation») sont à la fois décisives pour réussir une opération de référence garante de cohésion mais aussi particulièrement délicates à maîtriser.

\section{5. «This» pronom anaphorique encapsulant}

Finalement, nous examinerons le fonctionnement de «this» pronom, dont la caractéristique est de n'établir avec son référent qu'un lien sémantique, faute d'un terme déterminé permettant d'établir un lien lexical direct ou indirect comme dans les deux cas décrits précédemment. Il n'a pas de référent précis mais joue sur une reprise plus ou moins déterminée, voire ambiguë, qui peut aller de la reprise d'une proposition clairement énoncée, à la reprise d'un paragraphe ou même à celle d'un énoncé non formulé, mais néanmoins supposé partagé, entre l'énonciateur et le lecteur.

«This» a également ici une fonction résomptive mais son statut de pronom ne lui permet pas d'opérer la recatégorisation décrite précédemment, dans le cadre de ce que nous avons proposé d'appeler «anaphore "shifter" ». L'opération de déplacement, dans le cas précédent, est en effet assurée par le choix du terme déterminé par «this». Pour marquer la différence avec la fonction précédente, et l'accent mis sur la fonction de résumé plutôt que de déplacement, nous avons également emprunté à Francis (1994) le terme d'«encapsulant».

Voici un exemple extrait du corpus de sciences des matériaux langue 2 :

[Io] [Méthode] The last section of this work is a numerical study concerning the strain localisation patterns at the crack tip in f.c.c. single crystals under plane strain conditions at low temperature [Méthode_fin]. [Résultats_début] This has to be seen as a first step of a better understanding of the crack propagation process, having in view the competition between strain localisation models and fracture mechanics approach [Résultats_fin].

$\left(\mathrm{SM} \mathrm{L}_{2}\right)$

On voit la force du procédé qui permet de reprendre l'intégralité de l'étape précédemment décrite pour fonder l'ouverture de perspectives de recherche. Ici aussi, le choix du verbe modal et de la voix passive n'est pas anodin: «this» pronom permet clairement d'opérer un renversement de point de vue, l'auteur du texte prescrivant son interprétation au lecteur.

Ce second exemple, extrait du corpus de sciences des materiaux langue I, montre, au contraire, comment «this» pronom va venir cristalliser et appuyer la proposition de recherche en la résumant, assurant le passage de la proposition de recherche à la méthode. Le verbe fortement performatif («achieve») utilisé au présent passif pose comme acquise l'atteinte des objectifs.

[II] [Proposition_début] The objective of the present research is the creation of a numerical tool for the solution of general problems in three-dimensional linear-elastic fracture mechanics [Proposition_fin]. [Méthode_début] This is achieved with the 
integration of boundary element analysis and interactive computer graphic techniques [...] [Méthode_fin].

(SM LI)

Mais les limites du segment référentiel sont parfois difficiles à établir. C’est le cas dans l'exemple ci-dessous, extrait du corpus de didactique des mathématiques en langue i :

[12] [Résultats] In other words, many teachers found it difficult to use test results for planning their subsequent lessons. While they were able to analyze their students' work and could report in some detail on each student's performance, they failed to understand how this should affect their teaching [Résultats].

(DM LI)

Si la compréhension globale est parfaitement claire - les enseignants trouvent difficile d'établir le lien entre le travail d'analyse réalisé et leurs méthodes pédagogiques -, le segment référentiel ne peut être strictement délimité. Il peut inclure la proposition relative seulement («While they were able [...] performance»), ou intégrer également la proposition précédente, sans que l'interprétation en soit affectée. La cohérence est préservée: le flou dans la délimitation du segment référentiel fournit au lecteur une marge d'interprétation.

Cas similaire, dans le même corpus, mais cette fois le suivi du raisonnement est rendu plus complexe.

[I3] [Contexte] The emphasis needs to be placed upon the mental processes involved, and it is this which distinguishes mental computation from mental arithmetic, as defined in this study [Contexte].

(DM LI)

Le recours au procédé emphatique dans la phrase clivée: «it is this which», englobant l'opération de référence, en complexifie l'élucidation, tout en la mettant en valeur. On peut supposer que «this» reprend «the mental processes involved», mais on pourrait également imaginer que «this» englobe le fait de devoir mettre l'accent sur les processus mentaux. Ajoutons que l'utilisation du verbe «needs to be placed» indique une valeur prescriptive dont il est difficile de situer l'énonciateur, dans une forme proche du style indirect libre. Le procédé permet d'affirmer un point de vue tout en lui donnant un statut de vérité générale, visant à renforcer encore l'effet prescriptif.

L'ambiguïté dans l'établissement de la référence peut parfois devenir une difficulté dans les textes réalisés par des locuteurs francophones. Cet exemple est extrait du corpus de sciences des matériaux:

[I4] [Méthode] A probabilistic model in high cycle multiaxial fatigue is proposed [Méthode_fin]. [Contexte_début] This is based on the volumetric and energy based approach of the LAMEFIP and combined with the Weibull's formalism [Contexte_fin].

(SM L2) 
Pour que «this» encapsulant fonctionne de manière satisfaisante, il doit englober un segment précédent: dans ce cas, il doit s'agir de la proposition d'un nouveau modèle, ce qui revient à affirmer que c'est la proposition d'un modèle qui s'appuie sur une approche elle-même fondée sur l'énergie. La compréhension est loin d'être intuitive. On tend beaucoup plus spontanément à penser que c'est le nouveau modèle lui-même qui se fonde sur une approche particulière. Pourtant, si le rappel avait porté sur le modèle uniquement et non sa proposition, seuls «it» ou «this model» auraient établi un lien de cohérence satisfaisant.

Ce qui n'était qu'ambigu devient nettement problématique dans l'exemple ci-dessous, extrait du même corpus:

[I5] [Méthode] The second model allows simulating both crack growth rate and crack path, including crack bifurcation. This is a crystallographic continuous damage model [Méthode].

(SM L2)

La reprise du terme «model» lève les doutes possibles: il s'agit bien ici d'une erreur syntaxique. Seul «it» pouvait établir un lien satisfaisant en se substituant au terme «second model»: «this» pronom implique un englobement, rendu impossible par l'usage du verbe «to be» qui établit un lien d'identité. Cette erreur ne rend pas impossible la compréhension du raisonnement, mais compromet l'autorité du locuteur en déstabilisant la cohérence du texte.

Nous avons donc établi une typologie incluant quatre cas possibles. «This» exophorique établit le lien avec la situation d'énonciation, mettant l'accent sur la thèse comme résultat, ou sur le processus de recherche, ou sur le doctorant, avec diverses combinaisons possibles, selon l'élément sur lequel le locuteur choisit d'appuyer sa crédibilité.

«This» endophorique établit le lien intratextuel avec sa référence selon trois modalités. L'anaphore de reprise, comme son nom l'indique, reprend un terme antécédent mais en en modifiant l'environnement syntaxique. La place du référent et du terme déterminé dans les mouvements rhétoriques fournit une mesure du déplacement de focalisation effectué. L'anaphore «shifter» requalifie et englobe un élément d'information, qui peut être un seul terme. Le choix du terme déterminé établit la portée du déplacement réalisé, susceptible de permettre le passage d'un mouvement rhétorique à l'autre, à moins qu'il ne marque une étape interne à un mouvement rhétorique, par exemple d'un premier à un second résultat. Enfin, le pronom anaphorique encapsulant reprend et résume un élément d'information qui le précède pour en marquer le rôle dans la recherche. Ce procédé, puissant de par sa portée, est néanmoins fragile dans la mesure où le lien de référence ne peut être élucidé que sur des critères sémantiques, ce qui implique une bonne maîtrise des capacités d'interprétation du lecteur puisque l'énonciateur ne marque pas explicitement le point de référence dans la construction de son discours. 
La typologie que nous venons de proposer nous a permis de procéder, grâce à une base de données, à un relevé systématique des occurrences de «this» et de leur contexte syntaxique droit et gauche, et de stocker les résultats des différents paramètres de l'analyse de ces relevés.

\section{Discussion des résultats}

\subsection{Une palette d'utilisation variée au service de la construction d'une cohérence du texte, dans le cadre du genre}

L'étude des textes de notre corpus montre que toutes les fonctions que nous avons décrites peuvent être utilisées simultanément ou séparément pour construire un type de cohérence spécifique tout en contribuant à assurer le passage d'un mouvement rhétorique à l'autre. L'enchaînement stéréotypique est celui d'un «this» exophorique suivi soit d'un "this» anaphorique de reprise venant renforcer la cohérence d'une étape de la méthode, soit d'une anaphore «shifter» permettant d'assurer le passage de la proposition à la méthode ou le passage de la méthode aux résultats. Enfin, «this» pronom anaphorique encapsulant permet souvent d'établir le passage de la méthode aux résultats et notamment aux perspectives de recherche ultérieures. Il s'agit là de schémas récurrents mais ils sont loin de constituer une constante.

Nous donnons ci-dessous un exemple de texte présentant trois des quatre cas d'utilisation de «this» que nous avons définis:

[16] [Proposition_début] This study is an extension to the ongoing research on secondary mathematics teachers' knowledge. This study focused on the concepts of logarithms and logarithmic functions [Proposition_fin]. [Contexte_début] Several research studies have confirmed that high-school and undergraduate students have a very poor knowledge of logarithms and logarithmic functions. One of the possible reasons for students' difficulties could be an insufficient teachers' knowledge of this subject domain. As of yet, there has not been research into teachers' knowledge of logarithms. This study was an attempt to fill this gap. The deeper understanding of teachers' knowledge, particularly subject matter knowledge and related pedagogical skills, leads towards improvement of instructional approaches for more effective teacher training [Contexte_fin]. [Méthode_début] The questions posed in this study are: What do the designed tasks reveal about the nature of teachers' knowledge? What can be seen as the relationship between pre-service secondary mathematics teachers' subject matter knowledge tasks effective and useful as data collection tools for research in mathematics education [Méthode_fin]? [Résultats_début] This study identified that pre-service teachers are aware of possible difficulties of teaching or learning the concepts of logarithms and logarithmic functions. However, their insufficient subject matter knowledge disallowed participants to explain why the situations prompted by their own questions were indeed problematic and important. On the whole, the pre-service teachers' displayed a relatively weak content 
knowledge of logarithms and knowledge and related pedagogical content knowledge [Résultats_fin].

(DM Li; résumé cité ici dans son intégralité; Berezovsky, 2008: iii-iv)

La spécificité de ce texte est la répétition à cinq reprises de «this study», dont la fonction rhétorique est cependant soumise à des variations. La première occurrence correspond à un usage exophorique qui, grâce au choix du terme nominalisé «study», positionne le travail, présenté à la fois comme compte rendu et résultat d'une recherche. La deuxième occurrence peut être considérée comme une anaphore de reprise de la première et permet au texte de se projeter cette fois vers la proposition de recherche. La troisième peut également être considérée comme une anaphore de reprise et marque le passage au contexte théorique du projet. La quatrième a le même statut mais introduit la méthode sous la forme d'une problématique. Enfin, la dernière occurrence peut être considérée comme un encapsulant, englobant l'ensemble de la démarche exposée précédemment pour introduire la présentation des résultats. La fonction anaphorique de «this study», en construisant une chaîne de référence forte, souligne la cohérence du travail.

Les deux autres occurrences de «this» ont une fonction de «shifter». «This subject domain» reprend et requalifie la notion mathématique de fonction logarithmique, le «shifter» permettant le passage du point de vue scientifique au point de vue didactique. Quant à «this gap», il permet à l'auteur, en englobant et requalifiant toute la phrase précédente, de désigner ce que Swales (I990) appelle une «niche», démontrant ainsi la pertinence de son projet. Dans les deux cas, et à travers le maniement du lexique scientifique général, «this», en tant que «shifter», souligne les qualités interprétatives de l'auteur du texte et de la recherche qu'il présente.

On voit que la plasticité de «this» lui permet d'assurer des fonctions rhétoriques complémentaires, qui contribuent à la création d'une dynamique du texte conforme à une stratégie rhétorique particulière. C'est d'ailleurs pour pouvoir prendre en compte cette plasticité que nous avons choisi dans un premier temps de ne pas intégrer immédiatement dans notre étude la forme du pluriel «these». En effet, l'usage référentiel de «these» exclut les fonctions d'exophorique et d'encapsulant. Sa prise en compte nous semblait donc susceptible de fausser notre analyse du fonctionnement de «this» dans sa diversité d'usages rhétoriques. Il sera cependant intéressant de retourner ultérieurement vers une analyse de «these» pour affiner l'étude des usages anaphoriques de reprise et des «shifters».

La palette des usages endophoriques de «this» est particulièrement riche. La fonction exophorique insère le résumé dans son contexte d'énonciation et met en place une stratégie de présentation de la recherche selon qu'elle privilégie le lien avec la thèse, la recherche ou son auteur. La fonction endophorique se décline en trois possibilités selon qu'elle permet de reprendre un terme précédemment utilisé, de déplacer et requalifier un concept ou un élément d'information déjà évoqué, ou d'englober tout un passage du texte qui précède. Ces trois mécanismes ont pour point commun de déplacer la focalisation et donc de contribuer à diriger l'attention 
du lecteur au long des mouvements rhétoriques que nous avons désignés comme prototypiques de ce genre particulier (i.e. le contexte, la proposition, la méthode et les résultats).

Le premier facteur de réussite ou d'échec dans la réalisation d'un texte conforme aux objectifs du genre repose sur la capacité à utiliser ces fonctions de manière satisfaisante, en établissant une opération de référence et de déplacement de la focalisation correctement interprétable par le lecteur. Le choix d'un terme inadapté à l'opération de déplacement réalisée par le «shifter» rend problématique l'identification de la référence et compromet la cohérence du texte, déstabilisant l'efficacité de la démonstration et donc la construction de l'autorité de son auteur. C'est le cas dans l'exemple du «shifter» «this new variable» extrait du corpus de sciences des matériaux rédigé en langue seconde, et dont l'échec éventuel pourrait être lié à la difficulté d'établir un lien logique entre deux termes de langue scientifique générale, «variable» et «criterion», compte tenu de leur contexte collocationnel spécialisé («grain flow orientation»). En général, on note les difficultés que semblent éprouver les locuteurs non natifs à manier d'autres fonctions endophoriques de «this» que la fonction de reprise; ils ont en effet des difficultés à passer de la référence textuelle immédiate à la référence au concept sous-jacent à un discours, en choisissant les termes de langue scientifique générale du domaine susceptibles de reclassifier la référence. Au contraire, la capacité à manipuler les liens sémantiques hiérarchiques (l'hyperonymie en particulier) entre les termes, en démontrant la maîtrise du réseau terminologique spécialisé, est une marque d'autorité et de légitimité dans le domaine de spécialité.

Ce constat ouvre le champ d'une recherche spécifique portant notamment sur la capacité d'englobement des termes de langue de spécialité - «logarithms and logarithmic functions», "grain flow orientation» - par des termes dits de «langue scientifique générale» (Pecman, 2007) - «this subject domain», «this new variable», etc. -, et sur les difficultés que présente leur manipulation pour des locuteurs en seconde langue.

Le deuxième facteur de réussite ou d'échec implique les choix opérés parmi la gamme de possibilités de notre marqueur rhétorique et leur complémentarité. C'est là que se joue en partie l'efficacité de la stratégie rhétorique individuelle adoptée dans le cadre commun du genre.

Nous avons montré comment le déplacement de la focalisation s'opère de manière d'autant plus efficace qu'il est étayé par des choix lexicaux et syntaxiques conformes à l'opération rhétorique réalisée. La comparaison des stratégies rhétoriques de référence utilisées dans les deux disciplines prises en compte nous permettra maintenant de mieux comprendre comment le choix du type de déplacement de la focalisation au long des mouvements rhétoriques tend à s'adapter aux valeurs épistémologiques de la discipline concernée.

\subsection{Marqueurs du genre et stratégie disciplinaire}

Le relevé dans notre base de données des fonctions rhétoriques de «this» et l'étude de leur environnement nous permet en effet d'établir, au niveau des corpus, une analyse 
croisée des stratégies rhétoriques mises en œuvre dans les deux disciplines traitées ici, à savoir la didactique des mathématiques (DM) et les sciences des matériaux (SM).

Voici les résultats obtenus pour les fonctions endophoriques grâce à l'interrogation de la base de données que nous avons établie pour l'analyse:

- DM Li: 16 «shifters», 9 anaphores de reprise, 8 encapsulants;

- SM LI: 9 anaphores de reprise, 5 «shifters», 3 encapsulants;

- DM L2: 12 anaphores de reprise, 7 «shifters», 3 encapsulants;

- SM L2: I2 «shifters», 8 anaphores de reprise, 5 encapsulants.

Nous procéderons d'abord à une comparaison entre résumés de langue I puis tenterons d'interpréter le décalage relevé avec les textes rédigés en langue 2.

L'analyse des résumés de langue I en didactique des mathématiques fait apparaître une nette prédominance des fonctions anaphoriques «shifters» et encapsulantes qui assurent déplacement, englobement et requalification du segment référentiel. En revanche, les locuteurs anglophones en sciences des matériaux privilégient la fonction anaphorique de reprise.

Malgré la valeur numérique peu élevée des données, ce relevé paraît cohérent avec le contenu des résumés, tel qu'il se manifeste notamment à travers la répartition des mouvements rhétoriques. Des disparités apparaissent en effet, notamment en ce qui concerne la place accordée au mouvement définissant le contexte théorique ou institutionnel de la recherche, très développé en didactique des mathématiques et quasi absent en sciences des matériaux. Au contraire, le mouvement décrivant la méthode utilisée semble hypertrophié en sciences des matériaux.

À la lecture des résumés, la didactique des mathématiques apparaît comme une discipline dont l'objectif est de comprendre de manière aussi fine que possible un contexte pédagogique que l'on s'efforcera de modifier par l'action entreprise: d'où une prédominance du mouvement présentant le contexte mais aussi les résultats. Les projets présentés ont en effet le plus souvent une visée réformatrice.

Au contraire, les sciences des matériaux privilégient l'établissement d'un modèle qui, en représentant la réalité, va permettre de mieux la comprendre, pour la contrôler. Les résumés de thèse mettent donc l'accent sur la méthode suivie au détriment du contexte mais aussi, de manière plus surprenante, des résultats. En effet, contrairement à ce que l'on pouvait supposer, la discipline parait plus soucieuse de construire un modèle fiable de représentation de la réalité que de la modifier: il s'agit non pas de créer un nouveau phénomène mais de représenter un phénomène naturel, d'usure notamment, pour mieux le prévoir et le contrôler ensuite dans la réalité.

Dans ce contexte, les fonctions «shifter» et encapsulante paraissent en effet plus adaptées au modèle de la didactique des mathématiques, qui fonde la modification de la réalité sur les qualités interprétatives de la recherche. Alors que les sciences des 
matériaux, dont l'objectif est de construire un modèle de reproduction de la réalité solide et efficace, privilégieront l'anaphore de reprise, qui renforce d'abord l'effet de cohérence.

Dans cette mesure, l'utilisation des fonctions rhétoriques endophoriques confirme la distinction proposée notamment par Fløttum, Gedde-Dahl et Kinn (2006) entre sciences argumentatives, telles que la linguistique, et cumulatives, telles que la médecine. La didactique des mathématiques apparaît comme une science argumentative, fondant sa valeur sur la nouveauté de sa vision du domaine. Les sciences des matériaux se situent, elles, du côté cumulatif: chaque chercheur s'appuie sur les modèles construits antérieurement pour apporter une amélioration. On pourrait d'ailleurs émettre l'hypothèse que ce modèle explique la relative rareté des occurrences de «this», notamment dans leur valeur interprétative, dans le corpus de sciences des matériaux.

Aussi satisfaisante que paraisse cette lecture des données obtenues, force est cependant de constater qu'elle semble démentie par les résultats obtenus pour les résumés produits dans les mêmes disciplines par des locuteurs francophones, rédigeant en anglais. Constatation d'autant plus déstabilisante qu'on ne relève pas la même disparité en ce qui concerne la répartition des mouvements rhétoriques, identique dans les textes produits par des locuteurs anglophones et francophones.

Cependant, la prise en compte des données concernant l'environnement lexicosyntaxique de "this», dans le segment qu'il détermine et dans son segment référentiel, permet de mieux comprendre cette apparente contradiction. Ainsi, les doctorants francophones en didactique des mathématiques font de l'anaphore de reprise une utilisation fortement modalisée, mettant en avant les qualités de nouveauté et de pertinence des transformations proposées, là où les locuteurs de langue i favorisent les fonctions interprétatives de «this». En revanche, force est de constater que les francophones spécialistes de sciences des matériaux, qui privilégient la fonction de «shifter», échouent souvent dans le maniement de la fonction interprétative de «this», faute de savoir établir un lien satisfaisant entre le terme déterminé et sa référence, comme nous l'avons vu dans l'exemple [9].

Un des facteurs d'explication de ces spécificités des locuteurs non natifs de l'anglais pourrait être un défaut de maitrise linguistique: les didacticiens privilégiant l'anaphore de reprise, dont l'utilisation requiert moins de doigté que le «shifter» ou l'encapsulant; les spécialistes de sciences des matériaux échouant souvent dans leur volonté d'affirmer leurs qualités d'interprétation des données. L'existence du corpus français parallèle que nous avons constitué nous donnera la possibilité de vérifier ultérieurement cette hypothèse en procédant à une comparaison avec les procédés de détermination mis en œuvre dans la langue maternelle du doctorant.

Une autre piste d'explication s'appuierait non sur des facteurs linguistiques mais sur des facteurs culturels. La didactique des mathématiques, liée aux sciences de l'éducation, s'est développée différemment dans le monde anglo-saxon et francophone. Le domaine est particulièrement productif en Australie et Nouvelle-Zélande notamment et s'appuie fortement sur la théorie socioconstructiviste, dont des traces explicites figurent dans 
les résumés. En France, en revanche, on ne trouve pas de fondement théorique unique manifeste; l'approche semble plus pragmatique, ce qui peut expliquer une stratégie rhétorique fondée davantage sur la cohérence que sur l'interprétation. Cette hypothèse impliquerait que l'opposition habituelle entre des Anglo-Saxons plus pragmatiques et des Français plus cartésiens demande à être modulée en fonction des caractéristiques culturelles du modèle épistémologique qui fonde ce domaine de recherche.

En ce qui concerne les sciences des matériaux, on peut en revanche penser que le modèle académique général français tend à privilégier chez l'aspirant-chercheur des qualités interprétatives, venant contrebalancer l'importance accordée par la discipline dans son ensemble à la cohérence et à la solidité de la démonstration.

Bien entendu, le petit nombre de textes traités ne permet en aucun cas de tirer des conclusions définitives quant aux valeurs de la discipline concernée, surtout compte tenu de la double provenance linguistique et culturelle de leurs auteurs. Ce n'était d'ailleurs pas notre objectif. En revanche, l'analyse des fonctions rhétoriques de «this», lorsqu'elle prend en compte son environnement lexico-syntaxique, permet de mieux comprendre la gamme de possibilités dont dispose l'auteur du résumé pour mettre en œuvre une stratégie de communication adaptée à la fois aux objectifs du genre et aux valeurs de la discipline.

Les exemples traités dans notre première partie montrent qu'il est essentiel que soient pris en compte notamment, et comme on l'a vu dans nos exemples, le choix lexical du verbe (Kübler et Frérot, 2003) mais aussi le temps et la voix utilisés (voir exemple [2]). De même, la nature et la place des modalisants, selon qu'ils sont utilisés dans le segment référentiel ou dans le segment déterminé, modifieront la portée de la transformation introduite par l'usage de «this». Enfin, doit être prise en compte la manière dont est mise en ouvre la complémentarité des valeurs potentielles de «this».

Ainsi, un «this» exophorique de type «this study was conducted to» met d'emblée l'accent sur le processus de recherche et un scénario narratif privilégiant la valeur du travail d'expérimentation. En revanche, «this thesis addresses the internationally recognised issue of» pose un cadre interprétatif fort, cohérent avec un lien exophorique s'appuyant sur l'œuvre réalisée, et son auteur.

On ne peut donc limiter la réflexion à un simple comptage des différentes fonctions rhétoriques de «this» et à une analyse de leur répartition. L'élaboration de notre typologie aura en effet permis de mettre en évidence les facteurs complexes qui, dans l'environnement syntaxique de «this», en font un outil rhétorique particulièrement riche et modulable.

\section{Conclusion}

Les quatre fonctions de «this» que nous avons identifiées lui confèrent un rôle intéressant dans la construction de ce genre particulier qu'est le résumé de thèse, 
dont nous rappellerons que nous l'avons défini comme un type de discours susceptible de démontrer, à travers la description synthétique d'une recherche, l'aptitude de son auteur à intégrer la communauté des scientifiques et chercheurs. Nous avons notamment souligné le fait que le candidat doit à la fois prouver la cohérence de son projet de recherche, afficher sa légitimité face aux exigences spécifiques de la discipline et affirmer son autorité en apportant sa contribution particulière, conciliant ainsi conformité et capacité d'innovation. Ces valeurs sont diversement affirmées en fonction de la discipline. Il apparait en effet que ce genre s'inscrit dans l'espace commun défini d'une part par les attentes de l'institution universitaire dans son ensemble, et par les exigences des membres d'une communauté disciplinaire spécialisée d'autre part. Les auteurs des résumés de thèses adapteront donc l'usage qu'ils font de la diversité des fonctions de «this» à un type de stratégie rhétorique conforme aux objectifs du genre et de la communauté disciplinaire.

De manière générale, et en ce qui concerne la capacité de «this» à assurer la fonction de marqueur du genre, nous aimerions attirer particulièrement l'attention sur le fait que c'est notamment l'espace d'interprétation ouvert par l'opération de référence qui fonde la plasticité de «this» et en fait un dispositif rhétorique particulièrement précieux. Cet espace fournit en effet une marge d'interprétation que le lecteur saura ou non utiliser en fonction de ses compétences syntaxiques mais aussi disciplinaires. Les opérations d'encapsulation et de déplacement, notamment, parce qu'elles reposent sur des critères essentiellement sémantiques, impliquent que l'auteur du texte et son lecteur partagent un minimum de connaissances communes, en matière de manipulation du lexique scientifique spécialisé et général. De la capacité de l'auteur du résumé à partager un espace d'interprétation avec son lecteur, membre de la communauté spécialisée, dépend donc le fait que le texte réponde aux objectifs du genre, et contribue à créer une nouvelle voix de chercheur. L'étude que nous avons menée sur les usages de "this», en mettant au jour le rôle clé de l'espace d'interprétation commun au locuteur et au lecteur, nous semble ainsi offrir une entrée prometteuse pour l'examen du rôle des marqueurs de référence dans l'analyse du genre. Elle nous parait également ouvrir des possibilités d'application didactique immédiate, portant sur la prise de conscience du rôle des marqueurs de discours, notamment lorsqu'ils sont associés au vocabulaire de langue scientifique générale, pour exprimer des fonctions rhétoriques conformes au genre. Ce type d'application concerne tous les étudiants amenés à rédiger un discours scientifique en anglais, et particulièrement les étudiants francophones.

Bien entendu, «this» n'est qu'un des marqueurs possibles et ne constitue pas le seul dispositif capable de faire progresser la focalisation tout en assurant l'établissement de la référence. Nous noterons particulièrement l'usage de «it», dont nous avons vu qu'il faisait parfois l'objet d'une confusion avec «this», notamment chez les locuteurs non natifs. «It», qui implique l'élimination de toute ambiguïté dans l'établissement de la référence, à l'exception de son usage impersonnel, n'a pas la plasticité de «this» mais il présente un autre exemple intéressant d'évaluation de l'espace d'interprétation commun au locuteur et au lecteur dans un cadre spécialisé. C'est également le cas de 
certains usages de «the», notamment dans notre corpus de sciences des matériaux, dont la référence, externe au résumé, fait appel à des connaissances disciplinaires supposées partagées. Ces deux marqueurs offrent des pistes complémentaires d'étude des modalités de la réalisation d'un genre à travers la construction d'une chaîne de référence.

\section{Bibliographie}

Apotheloz, D. 1995. Rôle et fonctionnement de l'anaphore dans la dynamique textuelle. Genève : Droz.

Berezovski, T. 2008. Exposing Pre-service Secondary Mathematics Teachers' Knowledge through New Research Designed Methodology. Thèse de doctorat non publiée. Faculty of Education, Simon Fraser University. Burnaby.

BIBER, D. 2006. University Language: A Corpus-Based Study of Spoken and Written Registers. Studies in corpus linguistics 23. Amsterdam - Philadelphie: John Benjamins.

Biber, D., Connor, U. et Upton, T.A. 2007. Discourse on the Move. Amsterdam: John Benjamins.

BORDET, G. 2OII. Étude contrastive de résumés de thèse dans une perspective d'analyse de genre. Thèse de doctorat non publiée. Université Paris Diderot-Paris VII. Accessible en ligne sur TEL (thèses-en-ligne) : http://tel.archives-ouvertes.fr/tel-00650637/fr/.

Carter-Thomas, S. 2008. Teaching Coherence through Genre. Actes du colloque "De la France au Québec: l'écriture dans tous ses états». Colloque de Poitiers, IUFM PoitouCharentes, le I2-Is novembre 2008. Accessible en ligne à l'adresse suivante: http://hal. archives-ouvertes.fr/halshs-00385250 (consulté le 30/06/20II).

Charles, M. 2007. Reconciling Top-Down and Bottom-Up Approaches to Graduate Writing: Using a Corpus to Teach Rhetorical Functions. Journal of English for Academic Purposes 6 (4): 289-302.

Cornish, F. 20io. Anaphora: Text-Based or Discourse-Dependent? Functionalist vs. Formalist Accounts. Functions of Language I7 (2): 207-24I.

Fløttum, K., Gedde-Dahl, T. et Kinn, T. 2006. Academic Voices. Pragmatics \& beyond I48. Amsterdam - Philadelphie: John Benjamins.

Francis, G. 1994. Labelling Discourse: An Aspect of Nominal-Group Lexical Cohesion. In M. Coulthard (ed.), Advances in Written Text Analysis. Londres - New York: Routledge: 83-Ior.

GLEDHILL, C. 1994. La phraséologie et l'analyse des genres textuels. L'exemple des formules rhétoriques dans Le Monde. Aston Papers in Language Studies and Discourse Analysis 2. Accessible en ligne à l'adresse suivante: http://stl.recherche.univ-lillez.fr/sitespersonnels/ gledhill/Phraseologie_et_analyse_des_genres_textuels_GLEDHILL.pdf (consulté le 30/06/20II).

GLEDHILL, C. 1997. Les collocations et la construction du savoir scientifique. Anglais de spécialité I5 (I8): 85-104.

Halliday, M.A.K. 2004. The Language of Science. J. Webster (ed.). Collected works of M.A.K. Halliday 5. New York - Londres: Continuum International Publishing Group. 
Halliday, M.A.K. et Hasan, R. 1976. Cohesion in English. English language series 9. Londres: Longman.

Holzem, M. 200o. Le résumé de thèse: un exemple de reformulation explicative originale dans l'ensemble de la production des textes scientifiques. In P. Anderson, A. ChauvinVileno et M. Madini (eds), Répétition, altération, reformulation. Actes du colloque de Besançon, 22-24 juin 1998. Besançon: Presses universitaires franc-comtoises: 203-2II.

Hyland, K. 200o. Disciplinary Discourses: Social Interactions in Academic Writing. Applied linguistics and language study. Harlow - New York: Longman.

Hyland, K. 2002. Authority and Invisibility: Authorial Identity in Academic Writing. Journal of Pragmatics 34 (8): IO9I-III2.

Hyland, K. 2004. Graduates' Gratitude: The Generic Structure of Dissertation Acknowledgements. English for Specific Purposes 23 (3): 303-324.

KüBlER, N. et Frérot, C. 2003. Verbs in Specialised Corpora: From Manual Corpus-Based Description to Automatic Extraction in an English-French Parallel Corpus. UCREL Technical Papers 16 : 429-438.

Pecman, M. 2007. Approche onomasiologique de la langue scientifique générale. Revue française de linguistique appliquée I2 (2): 79-96.

Rastier, F. 2005. Enjeux épistémologiques de la linguistique de corpus. In G. Williams (ed.), La linguistique de corpus. Rennes: Presses universitaires de Rennes: 3I-45.

Risselada, R. et Spooren, W. 1998. Introduction: Discourse Markers and Coherence Relations. Journal of Pragmatics 30 (2): $\mathrm{I} 3 \mathrm{I}-\mathrm{I} 333$.

Sinclair, J.M. 2005. Language as a String of Beads. In E. Tognini-Bonelli et G. Del Lungo Camiciotti (eds), Strategies in Academic Discourse. Studies in corpus linguistics 19. Amsterdam - Philadelphie: John Benjamins: I63-168.

Swales, J.M. 1990. Genre Analysis: English in Academic and Research Settings. Cambridge applied linguistics series. Cambridge: Cambridge University Press.

Swales, J.M. et FEAK, C.B. 2009. Abstracts and the Writing of Abstracts. The Michigan series in English for academic \& professional purposes. Ann Arbor: University of Michigan Press.

Tutin, A. 2007. Autour du lexique et de la phraséologie des écrits scientifiques. Revue française de linguistique appliquée I2 (2): 5-I4. 